\title{
Circadian Biting patterns of Major Lymphatic Filariasis Vectors from Northwest Nigeria
}

\author{
Lawal $\mathrm{N}^{1,3}$, Idoko $\mathrm{AS}^{1}$, Abdullahi $\mathrm{H}^{2}$ and Imam $\mathrm{AA}^{3 *}$ \\ ${ }^{1}$ Department of Biochemistry \& Molecular Biology, Federal University Dutsinma Katsina State, \\ Nigeria \\ ${ }^{2}$ Tertiary Education Trust Fund, Nigeria \\ ${ }^{3}$ Department of Biochemistry, Bayero University Kano, Nigeria
}

\section{Research article}

Volume 4 Issue 2

Received Date: July 14, 2020

Published Date: July 27, 2020

DOI: $10.23880 /$ phoa- 16000158

*Corresponding author: Imam AA, Department of Biochemistry \& Bayero University Kano, Nigeria, Email: aaimam.bch@buk. edu.ng

\section{Abstract}

The circadian biting pattern of mosquito vectors is an important determinant of malaria and lymphatic filariasis (LF) transmission. Good knowledge of the vector host-seeking behavior, its outdoor/indoor biting preference, and nocturnal biting time is essential component for vector control strategies in Northwest Nigeria. The aim of this study was to assess the circadian biting time and identify the mosquito composition morphologically and molecularly. Mosquitoes were collected outdoor by human landing catches (HLC) using mouth aspirator from Batagarawa town and Gajerar Giwa village. Mosquitoes were morphologically and molecularly identified as Anopheles gambiae (M and S forms), Anopheles funestus s.s and Culex quinquefasciatus. The peak biting time for Culex quinquefasciatus were relatively similar across the study communities and the nocturnal biting rates showed an increased night time peak between $11 \mathrm{pm}$ to $12 \mathrm{am}$. However, the peak biting time of Anopheles gambiae s.l also varied between study communities and the nocturnal biting rates showed an increased night time peak between $9 \mathrm{pm}$ to $10 \mathrm{pm}$ and then $11 \mathrm{pm}$ to $12 \mathrm{am}$ but, the peak biting time for Anopheles funestus s.l which was found only in Gajerar Giwa village and the nocturnal biting rates showed an increased night time peak between 12am to 1am. This finding serves as a basis for creating an improved vector control strategies that target specific species, and if combine with other interventions could result in the elimination of malaria and LF transmission from Northwest Nigeria.

Keywords: Anopheles gambiae; Lymphatic Filariasis; Culex quinquefasciatus

Abbreviations: LF: Lymphatic Filariasis; HLC: Human Landing Catches; HBRs: Human Biting Rates; SSA: SubSaharan African; IRS: Indoor Residual Spraying; LLINs: Long Lasting Insecticidal Nets.

\section{Introduction}

Knowledge of the vector's biting behavior is an essential component in determining the vectorial capacity. The latter, in its classical form, comprises the parasite's extrinsicincubation period, the ratio of mosquitoes to humans, mosquito survival through one day, and human biting rates (HBRs) [1]. Sound knowledge of the vector's biting behavior is an important prerequisite to better understand and reduce malaria and lymphatic filariasis (LF) transmission. While endophagic species take their blood meal predominantly inside human dwellings, exophagic species feed mostly outdoors. Similarly, with regard to resting behavior, endophilic species commonly prevail indoors while exophilic species rest mostly outdoors. Anopheline species, such as Anopheles gambiae Patton 
Public Health Open Access

(Diptera: Culicidae) and Anopheles funestus Giles (Diptera: Culicidae), are known to prefer humans for their blood meal (anthropophilic), while others, such as Anopheles quadriannulatus Theobald (Diptera: Culicidae) and Anopheles melas Theobald (Diptera: Culicidae), feed predominantly but not exclusively on animals (zoophilic) [2]. Current malaria and LF vector control in sub-Saharan African (SSA) relies heavily on indoor insecticidal interventions using indoor residual spraying (IRS) and long lasting insecticidal nets (LLINs) [3-5]. The scale up of IRS and LLINs during the last decade has substantially reduced malaria and LF incidence in many parts of SSA $[6,7]$. These interventions reduce the density, feeding frequency and longevity of malaria and LF vectors by killing the vectors with insecticides or blocking their contact with humans $[8,9]$ and primarily target malaria and LF vectors that feed indoors and at night on sleeping humans [4]. The difference in the biting pattern contributes significantly in the transmission pattern of diseases among various hosts during different seasons. The rapid change in the ecosystem due to several factors such as the increase in global warming [10], unplanned urbanization, deforestation, changing human behavior [11], availability of hosts are found affecting the behavior of mosquitoes and thereby enhancing vector borne pathogens transmission [12]. This study was designed to monitor circadian biting patterns of Culicine and Anopheline mosquitoes as a function of seasonal variation in
Northwest Nigeria.

\section{Methods}

\section{Study Site and Human Landing Catches (HLC) Method}

Mosquitoes were collected using the Human Landing Catches (HLC) method [13]. Briefly, two collectors were made to sit in a dark room, with their legs exposed. Torch lights, switched on intermittently, were used to locate host-seeking mosquitoes. Landing mosquitoes that are ready to take a blood meal were then aspirated using a mouth aspirator. Trapped mosquitoes were then released into paper cups covered with mosquito netting and labeled to indicate the hour of collection (Figure 1):

- Batagarawa town, Batagarawa Local Government in the Sahel Savannah of Katsina State $\left(12^{\circ} 54^{\prime} 17^{\prime \prime} \mathrm{N}\right.$, $7^{\circ} 37^{\prime} 11^{\prime \prime} \mathrm{E}$ ), is a semi-urban area characterized by fewer of Anopheles gambiae s.l breeding sites.

- Sahel Savannah of Gajerar Giwa village $\left(12^{\circ} 95^{\prime} 21^{\prime} \mathrm{N}\right.$, $7^{0} 75^{\prime} 19^{\prime} \mathrm{E}$ ) in Rimi Local Government of Katsina State, where rice and vegetables irrigation are practiced using water from Ajiwa dam, characterized by large number of Anopheles funestus s.l breeding sites.

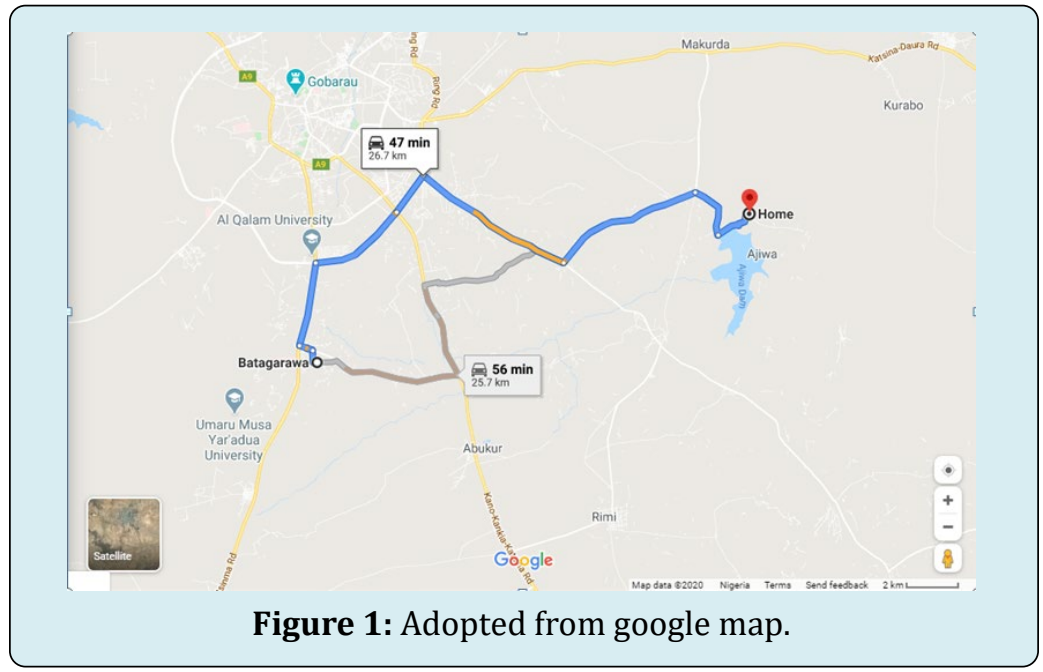

\section{Morphological Identification}

The working bench was cleaned with $70 \%$ ethanol. The mosquitoes brought from the study sites were separated using morphological features [14-17] to separate the mosquitoes into anophelines (Anopheles mosquitoes) and culicines (Aedes, Culex, and Mansonia mosquitoes). Each mosquito was picked with forceps into a petri dish and examined under a dissection (stereo) microscope (Olympus SZ60). All Anopheles mosquitoes were identified using the pale and dark band patterns on the costal margins of their wings. All culicines are also grouped based on the absence of the pale and dark band patterns on the costal margins of their wings and into their respective genera using morphological identification keys. In all genera of mosquitoes, the males were distinguished from the females by the presence of their plumose (bushy) antennae. The different species that had been morphologically identified were recorded on appropriate entomological data recording forms/sheets [18]. 


\section{DNA Extraction from Mosquitoes Collected from Study Sites}

Genomic DNA was extracted by Livak [19] protocol with little modification from the carcasses of mosquitoes after homogenizing with sterile plastic pestles in $100 \mu \mathrm{l}$ bender buffer. The homogenate was incubated at $65^{\circ} \mathrm{C}$ for $30 \mathrm{~min}$, followed by the addition of $14 \mu \mathrm{l} 8 \mathrm{M}$ K-acetate (to final concentration of $1 \mathrm{M}$ ). The mixture was vortexed and centrifuged at $14,000 \mathrm{rpm}$ for $20 \mathrm{~min}$ at $4^{\circ} \mathrm{C}$ using Eppendorf® Centrifuge 5415C. The supernatant was transferred into new 1.5ml Eppendorf tube. Two hundred microlitres of $100 \%$ ethanol were added to the supernatant. The mixture was vortexed and centrifuged at $14,000 \mathrm{rpm}$ for $15 \mathrm{~min}$ at $4^{\circ} \mathrm{C}$ and this was incubated at $-40^{\circ} \mathrm{C}$ for an hour, spun at $10,000 \mathrm{rpm}$ for $10 \mathrm{~min}$ and supernatant poured off. The pellet was then rinsed with $100 \mu$ l of $70 \%$ ethanol, spun at $10,000 \mathrm{rpm}$ for $5 \mathrm{~min}$. The supernatant was discarded and dried-up the pellet by leaving the tubes open on bench for an hour. The pellet was suspended in $100 \boldsymbol{\mu l} \mathbf{d H}_{2} \mathrm{O}$ then incubated at $65^{\circ} \mathrm{C}$ for $10 \mathrm{~min}$.

\section{Molecular Identification of Anopheles Mosquito Species}

Extracted DNA from morphologically identified mosquitoes was used as DNA templates for PCR. Each PCR reaction mixture of $25 \mu$ l contained $1 \times$ PCR buffer (Sigma, USA), $200 \mu \mathrm{M}$ each of the four deoxyribonucleotide triphosphates (dNTPs), $10 \mu \mathrm{M}$ each of the forward and reverse oligonucleotide primers, and 0.125units of Taq Polymerase enzyme (Sigma, USA). One microlitre of the genomic DNA was used as template for the amplification reaction.

After DNA amplification, the PCR products were electrophoresed in $2 \%$ agarose gel containing $0.5 \mu \mathrm{g} / \mathrm{ml}$ ethedium bromide in $1 \times$ TAE buffer using a mini gel system
(BIORAD USA). Eight microlitres of each sample was mixed with $1 \mu$ l of $5 \times$ Orange $G$ loading dye and loaded into a well of the gel. The voltage of the power unit was set at $100 \mathrm{~V}$ and run for an hour. A photograph of the gel was obtained using a UV trans-illuminator (UPC, USA) and a Polaroid camera of film type 667 (Polaroid, USA). The sizes of the PCR products were estimated by comparison with the mobility of a 100 base pair molecular weight size marker (Sigma).

\section{Molecular Identification of Culex quinquefasciatus}

Extracted DNA from morphologically identified Culex mosquitoes was used as DNA templates for PCR in which $5 \mu \mathrm{l}$ of extracted DNA was added to $1.5 \mathrm{ml}$ eppendorf tube then followed by acepip (F) $0.4 \mu \mathrm{l}$, ace gun (F) $0.8 \mu \mathrm{l}$ and B12465 (B) $0.8 \mu \mathrm{l}$ and $2 \mu \mathrm{l}$ of $1 \mathrm{x}$ Tag A, $0.1 \mu \mathrm{l}$ of $\mathrm{MgCl}, 11.92 \mu \mathrm{l}$ of $\mathrm{dH}_{2} \mathrm{O}$ and $0.1 \mu \mathrm{l}$ of Kappa Tag were added to the reaction mixture to form a total reaction volume of $20 \mu l$. The cycling conditions used were $95^{\circ} \mathrm{C}$ for $5 \mathrm{~min}$ followed by 40 cycles of denaturation at $94^{\circ} \mathrm{C}$ for $30 \mathrm{sec}$, annealing at $54^{\circ} \mathrm{C}$ for $30 \mathrm{sec}$ and extention at $72^{\circ} \mathrm{C}$ for $10 \mathrm{~min}$ then a final extention at $72^{\circ} \mathrm{C}$ for $10 \mathrm{~min}$. Electrophoresis using $1.5 \%$ agarose gel stained in ethidium bromide was run for $30 \mathrm{~min}$ after loading $3 \mu \mathrm{lCR}$ product.

\section{Results}

\section{Mosquito Sampling and Identification}

From the study communities, 50 Anopheles gambiae s. $l$ complex were randomly selected for molecular specie identification. It was observed that $25(50 \%), 3(6 \%)$ and $16(32 \%)$ in Figure 2 were Anopheles coluzzii (formerly M form), Anopheles arabiensis and Anopheles gambiae s.s (formerly S form) respectively. However Anopheles Funestus s.l. collected, 50 were randomly selected for molecular identification.

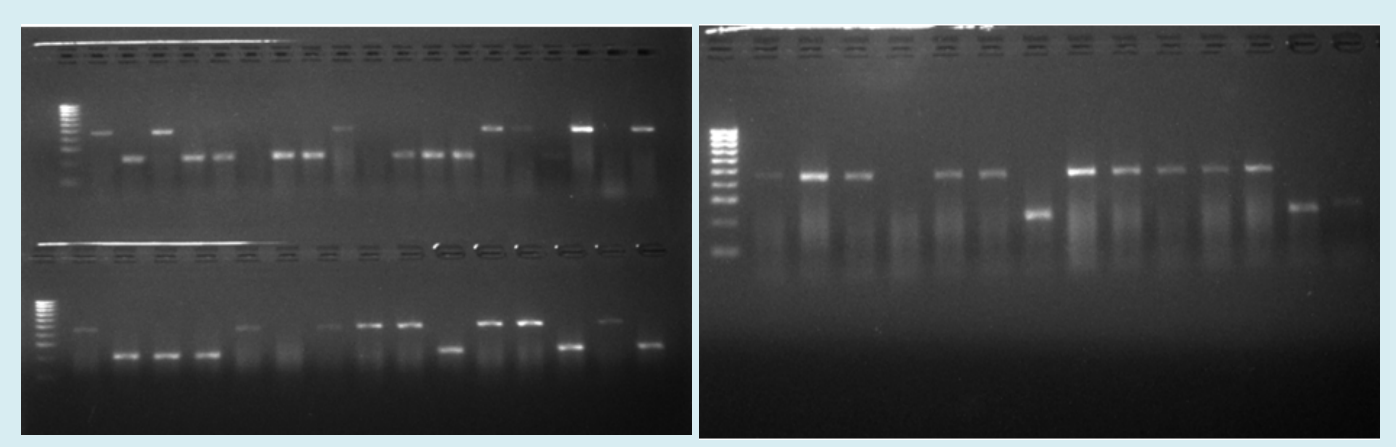

Figure 2: Gel electro pho re gram for the identification of members of the An. gambiae s.l complex. Lane M=100bp molecular weight marker, Lane 1,3,8,12,13,15,16,17,21,23,24,25,27,28,30 at 470bps=Anopheles coluzzii (formerly M form), Lane 2,4,5,6,7,9,10,11,14,23,26,28 at 240bps=Anopheles gambiae s.s (formerly S form), Lane 18-20 at 220bps=Anopheles arabiensis. 
Out of these $43(86 \%)$ in Figure 3 were predominantly Anopheles Funestus s.s from Gajerar Giwa village. Out of the large number of Culex spp that were morphologically identified, 50 Culex mosquitoes were randomly selected for molecular specie identification. The results showed $47(92.5 \%)$ in Figure 4 were predominantly Culex quenquifasciatus from the study communities.
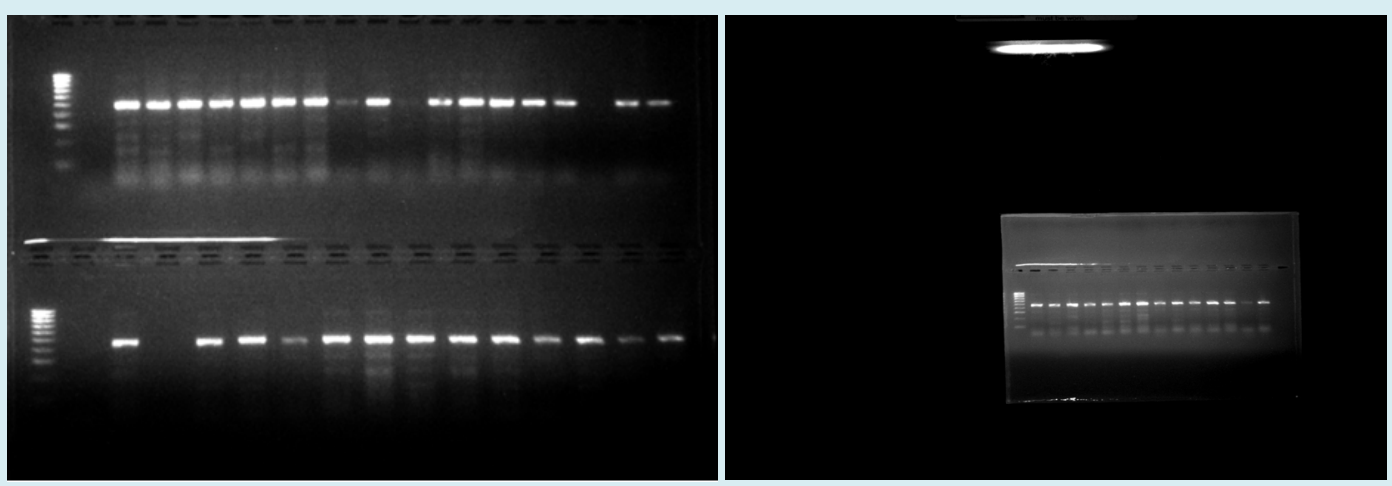

Figure 3: Gel electrophoregram for the identification of members of the Anopheles Funestus s.l complex. Lane M=100bp molecular weight marker, Lane $1-48$ at $460 \mathrm{bps}=$ Anopheles Funestus s.s.
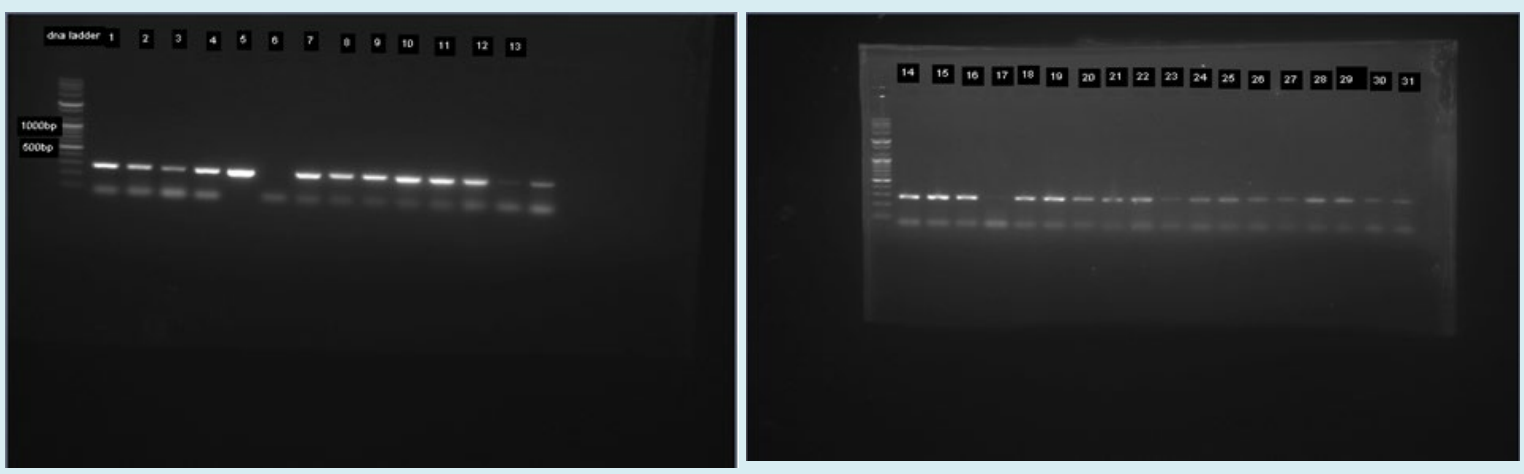

Figure 4: Gel electrophoregram for the identification of members Culex spp complex. Lane M=100bp molecular weight marker, Lane $1-31$ at 274 bps=Culex quenquifasciatus.

\section{Circadian Biting Time}

The peak biting times for Culex spp were relatively similar across the study communities Figure 5, with showing a unimodal distribution. The nocturnal biting rates showed an increased night time peak between $11 \mathrm{pm}$ to $12 \mathrm{am}$.

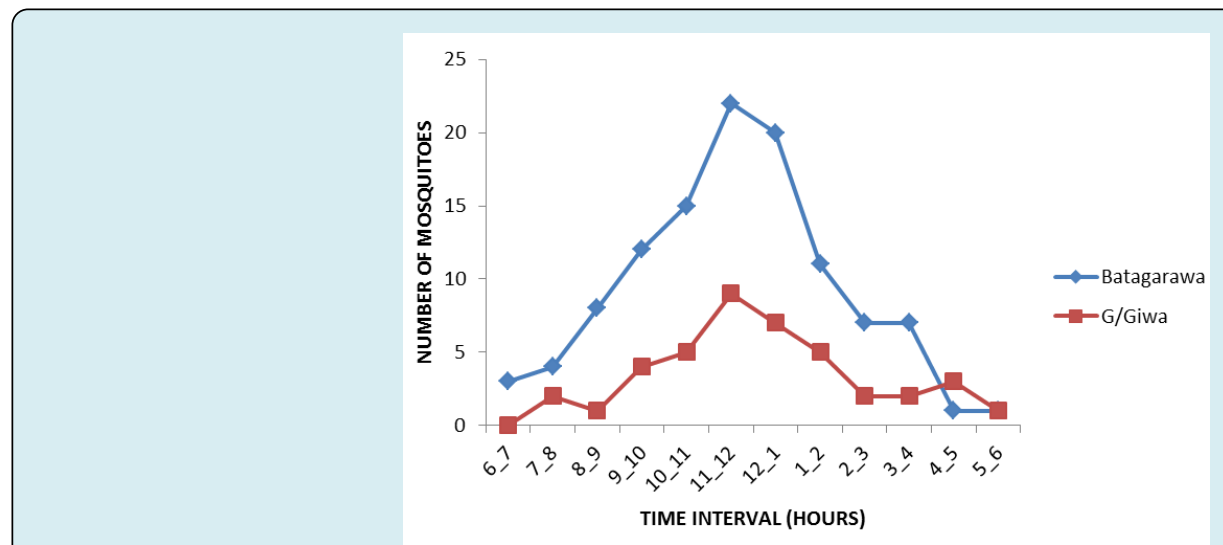

Figure 5: Circadian biting pattern of Culex quinquefasciatus at the study communities, from 6pm to 6am. 
However, the peak biting time of Anopheles gambiae s.l also varied between study communities Figure 6, with showing bimodal distributions. The nocturnal biting rates showed an increased night time peak between $9 \mathrm{pm}$ to $10 \mathrm{pm}$ and then $11 \mathrm{pm}$ to $12 \mathrm{am}$ but, the peak biting time for Anopheles funestus s.l which was found only in Gajerar Giwa village Figure 7, with showing single distribution. The nocturnal biting rates showed an increased night time peak between $12 \mathrm{am}$ to $1 \mathrm{am}$.

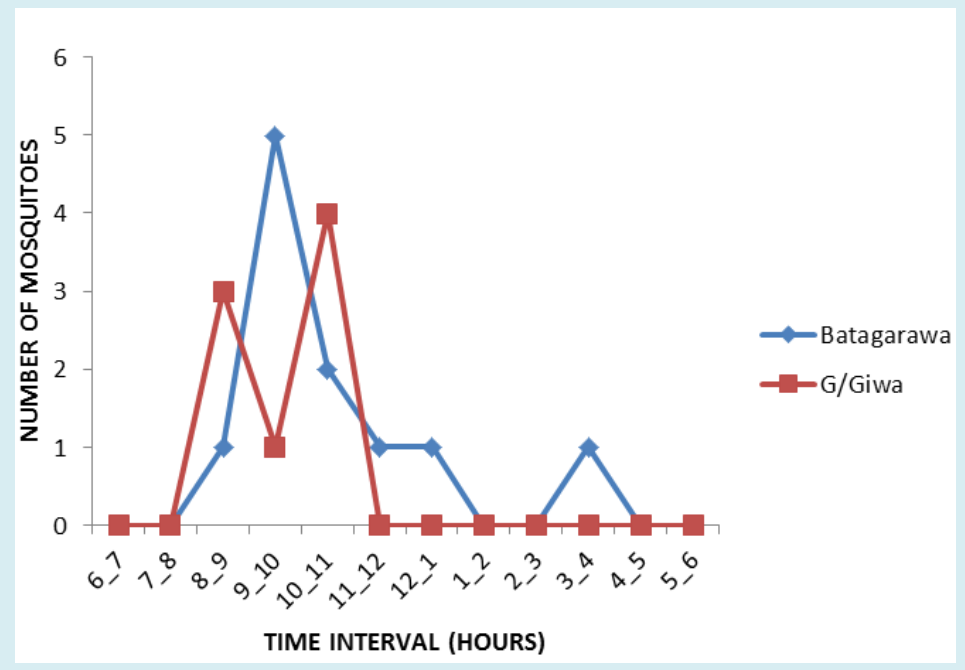

Figure 6: Circadian biting of Anopheles gambiae s.l at the study communities, from 6pm to $6 \mathrm{am}$.

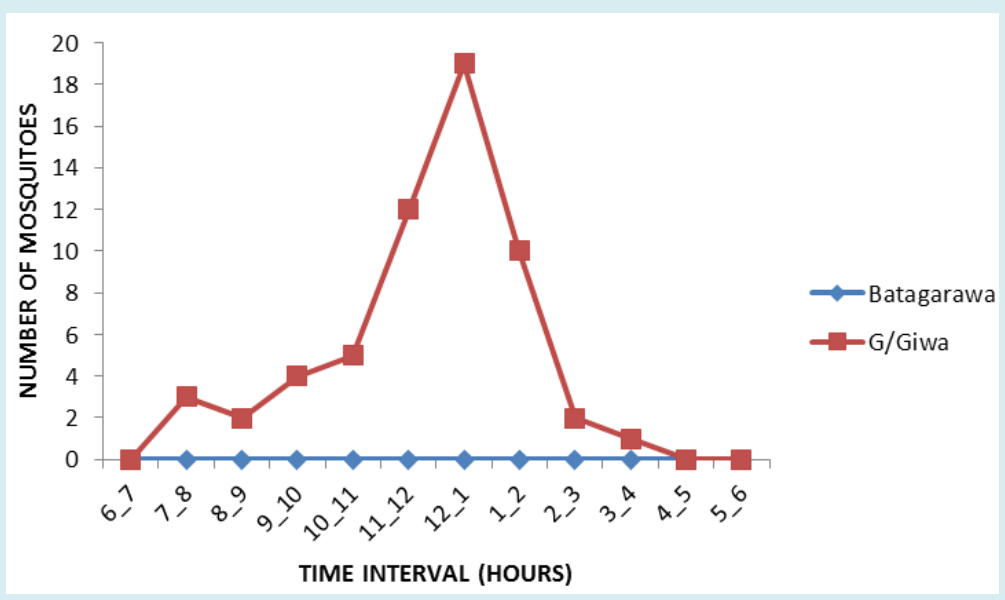

Figure 7: Circadian biting of Anopheles funestus s.l at the study communities, from 6pm to $6 \mathrm{am}$.

An important observation is that in most sampling communities, $70 \%$ or more of mosquitoes were collected between $5 \mathrm{am}$ to $6 \mathrm{am}$ in the morning. The outdoor biting pattern of the mosquitoes appeared to exceed the indoor biting pattern in both study communities.

\section{Discussion}

This present study indicated that Anopheles funestus s.s recorded $56.5 \%$ was the most abundant vector species caught in only Gajerar Giwa village. But Culex quinquefasciatus recorded $21.3 \%$ was caught from all the study communities followed by Anopheles gambiae s.l complex (13.4\%) in which (50\%) Anopheles coluzzii (formerly M form), (6\%) Anopheles arabiensis and (32\%) Anopheles gambiae s.s (formerly S form). These observations are corroborated by reports from Dar es Salaam by Mwakitalu E, et al. [20]. The higher abundance of Anopheles funestus s.s complex in the present study might be because mosquitoes were collected during the rainy season in which the Ano. Funestus s.s population was very high. The observed Anopheles Funestus s.s abundance has important implications in the transmission of both 


\section{Public Health Open Access}

malaria and lymphatic filariasis. It is therefore likely that the incline in Anopheles funestus s.s abundance observed in this present study will have comparatively more impact on the transmission of lymphatic filariasis than that of malaria.

In this study, three important lymphatic filariasis vectors in Northwest Nigeria were molecularly identified. Culex quinquefasciatus, Anopheles gambiae (M and S forms) and Anopheles funestus s.s were found to be the three humanbiting mosquitos' species occurring outdoors in the study area. The occurrence of the majority of biting is the early to middle phase of the night (during $09.00 \mathrm{pm}$ to $01.00 \mathrm{am}$ ) and peak biting by Culex quinquefasciatus between $11.00 \mathrm{pm}$ and $12.00 \mathrm{am}$ is contrary with the already known time of peak biting by the majority of human-biting Culex quinquefasciatus which showed peak biting after midnight in West Africa [21] and East Africa where it is an established vector [22]. This study was done outdoors during the time the people usually stayed outdoors (up till 12.00am) and inside (12.00am$06.00 \mathrm{am}$ ) when they were normally indoors. It is known that abundance, attractiveness, and availability of the human host are influencing vector biting behavior [23]. The peak biting time of Anopheles gambiae varies between Batagarawa town and Gajerar Giwa village and the nocturnal biting rate peak between $9.00 \mathrm{pm}-10 \mathrm{pm}$ and $10.00 \mathrm{pm}-11.00 \mathrm{pm}$ respectively. This is consistent with the already established time of peak biting by the majority of human-biting microfilaria positive Anopheles gambiae, i.e. between $11.00 \mathrm{pm}$ and $05.00 \mathrm{am}$ hours, a period when most people are in bed and under nets if they have them $[24,25]$. An. gambiae biting rate was moderate within the first 2 hours after dusk and increased quickly towards a 6-hours period of high biting. This finding is new and contrasts with many other African research sites, where the respective main vectors showed higher variation in nocturnal biting activity, featuring pronounced biting peaks and periods of lower activity.

Kenea 0, et al. [26] observed an early biting peak between $7.00 \mathrm{pm}$ and $8.00 \mathrm{pm}$ hours for the most important vector Anopheles ziemanni Grunberg (Diptera: Culicidae) in Ethiopia, while An. arabiensis showed two peaks, from $8.00 \mathrm{pm}$ to $10.00 \mathrm{pm}$ hours and around midnight. Similarly, a study from Ethiopia [27] found An. gambiae s.l. and mosquitoes from the Anopheles coustani complex to have two activity peaks, one between dusk and $10.00 \mathrm{pm}$ hours and a smaller in the early morning around 04.00am hours.

Anopheles funestus which was only found in Gajerar Giwa village and this could be attributed to the presence of more permanent water for breeding provided by a larger Ajiwa dam in this locality. Anopheles funestus is known to breed all year round and prefer permanent, stagnant water bodies such as shores of rivers and creeks, swamps or fish ponds for breeding, the peak biting rate showed an increase night time peak between $12.00 \mathrm{am}$ to $1.00 \mathrm{am}$. Similar scenario was also reported in Zimbabwe by Sande S, et al. [28] that, the peak biting time of Anopheles funestus was between $10.00 \mathrm{pm}$ and $11.00 \mathrm{pm}$ hours, and around 03.00am hours.

\section{Conclusion}

From the study communities there were high vectors of lymphatic filariasis outdoor biting activity throughout the nights with marked single or multiple peaks. These biting patterns could be the consequence of mosquito tolerance to the recently achieved high LLIN and IRS coverage in the study communities. Higher activities of mosquito vectors of lymphatic filariasis were observed throughout the study nights, vectors contact at any time during the nights are high when people are not sleeping under a bed net. Undertaking the goal of further reducing malaria and lymphatic filariasis transmission might raise the need for additional vector control strategies that either decrease vector populations or reduce human-vector contact.

\section{References}

1. Brady OJ, Godfray HC, Tatem AJ, Gething PW, Cohen JM, et al. (2016) Vectorial capacity and vector control: Reconsidering sensitivity to parameters for malaria elimination. Trans R Soc Trop Med Hyg 110(2): 107-117.

2. Sylvie M, Pierre C, Jean M (2008) Biodiversity of malaria in the world. John Libbey Eurotext, France, pp: 464.

3. Dambach P, Traore I, Becker N, Kaiser A, Sie A, et al. (2014) EMIRA: Ecologic malaria reduction for Africainnovative tools for integrated malaria control. Glob Health Action.

4. (2014) Control of residual malaria parasite transmission: Guidance note. World Health Organization.

5. Braack L, Hunt R, Koekemoer LL, Gericke A, Munhenga G, et al. (2015) Biting behavior of African malaria vectors: where do the main vector species bite on the human body? Parasites \& Vectors 8: 76.

6. D'Acremont V, Lengeler C, Genton B (2010) Reduction in the proportion of fevers associated with Plasmodium falciparum parasitaemia in Africa: a systematic review. Malar J 9: 240.

7. Mharakurwa S, Mutambu SI, Mberikunashe J, Thuma PE, Moss WJ, et al. (2013) Changes in the burden of malaria following scale up of malaria control interventions in Mutasa District, Zimbabwe. Malar J 12: 223.

8. Gimnig JE, Vulule JM, Lo TQ Kamau L, Kolczak MS, et al. (2003) Impact of permethrin-treated bed nets on the 
entomologic indicies in an area of intense year-round malaria transmission. Am J Trop Med Hyg 68(S 4): 115120.

9. Mutuku FD, King $\mathrm{CH}$, Mungai $\mathrm{P}$, Mbogo C, Mwangangi J, et al. (2011) Impact of insecticide-treated bed nets on malaria transmission indices on the south coast of Kenya. Malar J 10: 356.

10. Chaves LF, Cohen JM, Pascual M, Wilson ML (2008) Social exclusion modifies climate and deforestation impacts on vector-borne diseases. PLOS Neglected Tropical Diseases.

11. Kabbale FG, Akol AM, Kaddu JB, Onapa AW (2013) Biting patterns and seasonality of Anopheles gambiae sensu lato and Anopheles funestus mosquitoes in Kamuli District, Uganda. Parasites \& Vectors 6: 340-348.

12. Edman JD, Tylor DJ (1968) Culex nigripalpus: seasonal shift in bird-mammal feeding ratio in a mosquito vector of human encephalitis. Science 161(3836): 67-68.

13. (2003) Training module on community home-based prevention of disability due to lymphatic filariasistutor's guide. World Health Organization.

14. Gillies MT, De Meillon B (1968) The Anophelinae of Africa South of the Sahara (Ethiopian Zoographical Region). South African Institute for Medical Research, Mosquito Taxonomic Inventory 54: 343.

15. Danilov VN (1982) A Key for the Mosquitoes of the Genus Mansonia. An Inst Hig E Med Trop (Lisboa) 9: 31-36.

16. Highton RB (1983) Taxanomic keys for the identification of the Afrotropical mosquitoes. Taxanomic keys for the identification of the Afrotropical mosquitoes, pp: 1-82.

17. Gillies MT, Coetzee M (1987) A supplement to the Anophelinae of Africa South of the Sahara. South African Institute for Medical Research. Mosquito Taxonomic Inventory 55: 1-143.

18. Osei JHN (2013) Assessing Transmission of lymphatic filariasis using mosquitoes in communities with at least five rounds of mass drug administration in Ghana. International Household Survey Network.
19. Livak KJ (1984) Organization and mapping of a sequence on the Drosophila melanogaster X and Y chromosomes that is transcribed during spermatogenesis. Genetics 107(4): 611-634.

20. Mwakitalu E, Malecela MN, Pedersen ME, Mosha WK, Simonsen PE (2013) Urban lymphatic filariasis in the metropolis of Dares Salaam, Tanzania. Parasites \& Vectors 6: 286.

21. Subra R (1981) Biology and control of Culex pipiens quinquefasciatus (Diptera: Culicidae) with special reference to Africa. International Journal of Tropical Insect Science 4(1): 319-338.

22. Pedersen EM, Mukoko DA (2002) Impact of insecticidetreated materials on filarial transmission by the various species of vector mosquito in Africa. Anna Trop Med Parasitol 96(2): S91-S95.

23. Mokry JE (1980) A method for estimating the age of field-collected female Simulium damnosum s.1 (Diptera: Simuliidae). Tropenmed Parasitol 31(1): 121-127.

24. Maxwell CA, Wakibara J, Tho S, Curtis CF (1998) Malariainfective biting at different hours of the night. Med Vet Entomol 12(3): 325-327.

25. (1999) The control of malaria mosquitoes in Uganda. Guidelines Kampala: Ministry of Health, Uganda.

26. Kenea O, Balkew M, Tekie H, Gebre Michael T, Deressa W, et al. (2016) Human-biting activities of Anopheles species in south-central Ethiopia. Parasites \& Vectors 9: 527.

27. Taye B, Lelisa K, Emana D, Asale A, Yewhalaw D (2016) Seasonal dynamics, longevity, and biting activity of anopheline mosquitoes in southwestern Ethiopia. J Insect Sci 16(1): 6.

28. Sande S, Zimba M, Chinwada P, Masendu HT, Makuwaza A (2016) Biting behaviour of Anopheles funestus populations in Mutare and Mutasa districts, Manicaland province, Zimbabwe: implications for the malaria control programme. J Vector Borne Dis 53(2): 118-126. 